\title{
Lidil
}

Revue de linguistique et de didactique des langues

$57 \mid 2018$

Démarches créatives, détours artistiques et appropriation des langues

\section{Les problèmes de traduction dans le PISA : les limites de la standardisation des tests de compréhension}

Translation Problems in the PISA: Some Limits to Standardization of Literacy

Assessment

Daniel Bart, Bertrand Daunay et Chedia Belaid Mhirsi

\section{OpenEdition}

Journals

Édition électronique

URL : http://journals.openedition.org/lidil/4936

DOI : $10.4000 /$ lidil.4936

ISSN : 1960-6052

Éditeur

UGA Éditions/Université Grenoble Alpes

Édition imprimée

ISBN : 978-2-37747-048-8

ISSN : $1146-6480$

Référence électronique

Daniel Bart, Bertrand Daunay et Chedia Belaid Mhirsi, «Les problèmes de traduction dans le PISA : les limites de la standardisation des tests de compréhension », Lidil [En ligne], 57 | 2018, mis en ligne le 01 mai 2018, consulté le 22 avril 2019. URL : http://journals.openedition.org/lidil/4936 ; DOI : 10.4000/ lidil.4936

Ce document a été généré automatiquement le 22 avril 2019

(c) Lidil 


\title{
Les problèmes de traduction dans le PISA : les limites de la standardisation des tests de compréhension
}

\author{
Translation Problems in the PISA: Some Limits to Standardization of Literacy \\ Assessment
}

Daniel Bart, Bertrand Daunay et Chedia Belaid Mhirsi

\section{Introduction}

1 Parmi les importants problèmes méthodologiques que rencontrent les enquêtes comparatives internationales, la question de la traduction n'est pas la moindre : de fait, elle est l'un des arguments les plus récurrents et les plus fermes de ceux qui dénoncent l'«illusion comparative» (Guérin-Pace \& Blum, 1999). C'est la raison pour laquelle le Programme international de suivi des acquis des élèves (PISA), venu après de prestigieux autres programmes, a mis en place une réflexion d'envergure, qui a débouché sur des principes d'une grande solidité concernant le processus de traduction des items.

2 Nous nous proposons, dans cet article, d'interroger non ces principes, mais leur mise en cuvre effective, dans la mesure où il nous est apparu souvent que la réalisation concrète des traductions du PISA contrevient assez nettement à ces derniers. C'est cette contradiction entre l'affichage de principes et leurs transgressions effectives que nous souhaitons étudier et c'est dans cette optique que nous tenterons, après d'autres ${ }^{1}$, d'aborder les problèmes que pose le PISA dans son entreprise de traduction des items. Plutôt qu'un survol de quelques problèmes rencontrés dans une série d'exercices tirés de cette évaluation, nous avons fait le choix d'approfondir un exemple par l'analyse d'un exercice - ou unité - spécifique, "Macondo ${ }^{2}$ » (qu'on trouvera dans l'annexe 1). Cet exercice, donné une seule fois en 2000 (premier cycle du PISA), a été réutilisé pour un 
essai de terrain en vue de la préparation du cycle 2009, à l'occasion duquel il a été rendu public (en partie) en tant qu'exercice représentatif ${ }^{3}$. Le fait de ne choisir qu'un exemple, certes, amène à traiter un seul des domaines évalués par le PISA (la compréhension de l'écrit) et un seul type d'écrit parmi ceux que le PISA envisage dans ce domaine-là (en l'occurrence, un texte littéraire, qui, sans doute, pose des questions spécifiques), mais l'exemple est à prendre comme illustration de problèmes de portée plus générale, comme on le verra.

Précisons que notre démarche est à la fois descriptive - hors d'une logique d'évaluation ou de disqualification des processus de traduction du PISA - et critique, puisque nous visons à rendre compte de problèmes de traduction qui se posent au regard des principes édictés par ce programme lui-même. Dans un premier temps, nous rappellerons les principes de traduction du PISA tels que la littérature diffusée par le programme les présente. Nous verrons ensuite, par une analyse qualitative du test dans cinq langues (l'anglais, l'arabe, l'espagnol, le français, le portugais) ${ }^{4}$, les diverses manières dont les traductions du PISA enfreignent ces principes dans les faits et quelles sont les conséquences de ces choix de traduction ${ }^{5}$.

\section{Les précautions méthodologiques du PISA}

Les risques de disparité entre les pays et, partant, d'une validité moindre des résultats, ont donné lieu, de la part du PISA, dès son premier cycle en 2000, à des protocoles de traduction destinés à assurer une comparabilité du matériel de test par-delà les différences linguistiques, voire une équivalence sémantique aussi grande que possible (OECD, 2002, p. 57-70 ; voir aussi Grisay, 2003; Grisay, De Jong, Gebhardt, Berezner \& Halleux-Monseur, 2007). Le PISA cherchait par là à limiter les problèmes de traduction constatés dans des évaluations internationales précédentes (Rocher, 2003 ; Guérin-Pace \& Blum, 1999), problèmes que l'accroissement du nombre de pays appelés à participer au programme ne pouvait qu'accentuer.

5 Il vaut la peine de présenter ici quelques-uns des principes développés, avec une prudence méthodologique assez remarquable, qui contrastent, comme on le verra plus loin, avec la réalité des traductions étudiées. Parmi ces procédures d'assurance qualité de la traduction (OECD, 2002 p. 57), la littérature du PISA insiste tout d'abord sur le fait que le matériel du test (exercices, guides de correction, etc.) est disponible initialement dans deux langues sources (anglais et français, langues officielles de l'OCDE). Cette décision se veut un moyen de limiter le poids implicitement donné à une langue de référence unique (ibid., p. 59), généralement l'anglais dans les évaluations antérieures - même si l'on s'en tient à deux langues « indo-européenne » (ibid.).

Cela ne concerne cependant pas les manuels opérationnels du PISA fournis uniquement en anglais (OECD, 2014, p. 90), ainsi que les unités des domaines d'évaluation testés épisodiquement comme la compréhension de textes électroniques lors du PISA 2009 (OECD, 2012, p. 36) ou la littéracie financière pour le PISA 2012 (OECD, 2014, p. 90). Plus largement, le poids de l'anglais reste prédominant: la majorité des 37 unités de compréhension de l'écrit du PISA 2000 a été proposée en anglais (OECD, 2002, p. 262-265) et les versions françaises des quelques unités initialement proposées dans une autre langue (p. ex. le japonais ou le danois) semblent avoir été produites non au moyen d'une traduction depuis leur langue d'origine, mais à partir de leur version traduite en anglais (Grisay, 2003, p. 230). Au-delà des questionnements linguistiques et culturels que suscite 
ce choix méthodologique, il interroge la notion de « version source » promue par le PISA : la plupart des unités dites sources en français sont déjà des traductions depuis des versions anglaises, certaines étant elles-mêmes issues de traduction.

7 À partir de ces deux sources, dont le PISA dit tester statistiquement l'équivalence (OECD, 2002, p. 64-67), chaque pays est supposé produire la ou les versions nationales qui lui seront nécessaires pour évaluer les élèves dans leur(s) langue(s) d'enseignement. Pour ce faire, le PISA a fixé le principe d'une double traduction des versions sources selon un processus complexe. Ce processus devrait, autant que faire se peut, impliquer pour chacune des versions sources, au niveau national, deux traductions indépendantes conciliées à travers l'intervention d'un troisième traducteur superviseur. Lorsque cette version validée nationalement ne concerne pas une langue d'enseignement rare ou spécifique, elle doit ensuite être elle-même révisée au niveau international par une équipe de traducteurs pour établir la version finale.

8 Soulignons cependant que malgré ces principes, le PISA a pu s'appuyer sur des traductions issues du commerce pour certaines unités (comme nous le verrons pour «Macondo» dans la suite de cet article), ou que l'agenda a pu contraindre les experts français à commencer leur traduction des versions sources anglaises avant que celles-ci ne soient achevées (OECD, 2002, p. 60), tandis que certains pays semblent avoir utilisé des procédures de traduction alternatives (ibid., p. 67-69).

9 Mais on voit aussi à l'œuvre dans la présentation de ces principes toute une série de précautions qui vont jusqu'au détail le plus fin. Ainsi, dans le cadre de la formation et de la coordination des traducteurs, le PISA diffuse des directives (OECD, 2004) qui insistent par exemple sur le respect de la mise en forme des unités (typographie, taille des illustrations) tout autant que sur des contenus à viser: possibilité d'adapter ou pas les noms de lieux, d'unités de mesure ou de monnaies, conseils d'utilisation de tournures lexicales ou syntaxiques appropriées aux contextes nationaux ou encore attention à porter aux connecteurs, à la longueur des phrases ou à la formulation des QCM. En outre, le matériau à traduire peut être assorti de remarques et annotations précises destinées à éviter toute ambigüité.

10 Ces principes, rappelons-le, visent à obtenir la plus grande équivalence sémantique possible, laquelle est également contrôlée par des essais de terrain qui cherchent à éliminer les unités qui auraient des fonctionnements trop marqués localement (par exemple des exercices qui obtiendraient des taux de réussite nuls dans certains contextes nationaux ou culturels) ou par des calculs d'indices de lisibilité des différentes versions des textes (OECD, 2002).

11 On le voit donc: le PISA ne ménage pas ses efforts dans son importante entreprise de traduction, ce qui explique qu'au fur et à mesure du déroulement du programme, il rende compte d'évolutions apportées aux démarches de traduction pour résoudre certains problèmes (voir par exemple OECD, 2016a, p. 8 et 2016b, p. 1-2). Pourtant, il faut noter la résistance du PISA à l'égard de certaines difficultés qui persistent. Ainsi, les questionnements et les débats que pose le premier rapport technique du PISA 2000 (OECD, 2002) sont moins présents dans les éditions suivantes, à notre connaissance. Observons cet exemple : ce premier rapport montre que la traduction tend à accroitre le nombre de mots et de caractères ; $s$ 'il assure que les contrôles statistiques effectués permettent de ne pas supposer d'effet significatif sur la réussite, il admet que « l'hypothèse d'un effet sur la performance des élèves ne peut être écartée » (ibid., p. 66, nous traduisons) ; l'expression 
de cette prudence méthodologique (répétée encore dans le rapport technique suivant: OECD, 2005, p. 70), ne semble ensuite plus apparaitre dans la littérature du PISA...

Le programme ne semble pas non plus enclin à se faire l'écho des travaux d'experts ayant contribué au PISA et tout particulièrement à ceux qui concernent la traduction, quand bien même ils auraient suscité une amélioration des procédures (par exemple les travaux qui interrogent les liens entre les résultats et les caractéristiques linguistiques et culturelles des pays participants: Grisay, De Jong, Gebhardt, Berezner \& HalleuxMonseur, 2007, p. 265 ; Rocher, 2003, p. 133).

Ces questions dépassent bien sûr la question de la traduction et touchent aussi au format des unités, des questions, à la proximité avec les programmes et pratiques scolaires, etc. Mais cela permet peut-être d'observer que les langues et tout ce qu'elles engagent offrent quelque résistance aux démarches qualité même les plus affutées. C'est ce que veut illustrer l'analyse d'un exemple précis, présentée ci-après.

\section{Des principes de traduction mis à mal : l'exemple de «Macondo »}

Rappelons que nous avons choisi d'analyser l'unité « Macondo » dans la mesure où elle est décrite avec précision dans le Cadre d'évaluation du PISA 2009 (OCDE, 2012, p. 166-171; OECD, 2009, p. 174-180) afin d'exemplifier la conception des tests d'évaluation de la compréhension de l'écrit (OCDE, 2012, p. 46; OECD, 2009, p. 47). Le texte qui concerne l'unité « Macondo » est extrait de Cent ans de solitude de Gabriel García Márquez (1967). D'après OECD (2002, p. 262), l'unité a été initialement proposée en espagnol. Cette version initiale a été ensuite l'objet d'une traduction en anglais et en français qui sont, nous l'avons vu, les deux langues de production des versions sources des unités du PISA.

15 Voici une première étrangeté : ces deux versions sources, n'ont pas été réalisées en suivant les principes scrupuleux du PISA que nous avons rappelés ci-dessus, mais proviennent, avec des aménagements mineurs, des traductions commerciales réalisées au moment de la parution de l'ouvrage, celle de Claude et Carmen Durand pour le français (qui date de 1968), celle de Gregory Rabassa pour l'anglais (1970). En matière de textes littéraires, le recours à des traductions déjà disponibles est en effet envisagé dans tous les Translation and adaptation guidelines, produits en vue de la réalisation du matériel de test (pour exemple, voir le dernier en date, concernant le cycle PISA 2018 : OECD, 2016c, p. 21).

Mais, alors que le texte original de García Márquez a été légèrement modifié dans la version espagnole du test PISA, pour des raisons de lisibilité du texte, il est troublant que les concepteurs du test délèguent, de façon assez aléatoire, ce travail d'adaptation dans les autres langues (y compris pour les deux versions sources). Cela est d'autant plus troublant que certains des choix faits par les traducteurs engendrent des distorsions entre les versions, du point de vue de la lisibilité, si l'on prend en compte les propriétés de chaque langue. C'est ce que nous allons voir dans le détail.

\subsection{Choix lexicaux}

Commençons par le lexique choisi. Dans le texte de García Márquez, on trouve le mot centavo, terme familier à un hispanophone - et à un lusophone ; rien d'étrange à ce qu'on trouve ce mot dans les versions espagnole et brésilienne du PISA. 
Mais pourquoi le retrouve-t-on dans le texte français et dans le texte anglais ?

Le public, qui payait deux centavos pour partager [...]

The audience, who paid two centavos apiece to share [...]

Ces mots surprennent dans ces deux langues et créent un effet d'exotisme qui n'existe pas dans le texte original. La version française s'est inspirée de la traduction des Durand, mais dans la traduction anglaise de Rabassa il n'y avait pas centavos, mais cents. Le PISA a donc, sur ce point, compliqué la traduction de Rabassa, qui est pourtant sa source... Pour cadrer avec ce choix, la version arabe tunisienne propose un équivalent phonétique de centavos, (/qui peut se prononcer /scntafus) سنتفوس6). En fait, la décision de conserver centavos procède d'une consigne donnée pour la traduction: «Si vous utilisez une traduction publiée [...] gardez le terme original "centavos" pour désigner la monnaie.» Cela n'a rien d'absurde et correspond même à un usage assez fréquent de ne pas traduire ce qui relève $\mathrm{du}$ domaine économique, mais n'est pas sans poser problème au regard des exigences d'une comparaison internationale des compétences de lecture des élèves.

Voici un autre exemple de choix lexical, là encore conforme à un certain usage en traduction, mais peu compréhensible dans ce contexte. Il concerne le maintien du don espagnol dans les versions anglaise et française (ou dom en portugais), alors que cela ne correspond pas à un usage en français (Monsieur aurait très bien fait l'affaire) et alors que Rabassa et Zagury, par exemple, avaient fait le choix de supprimer le titre.

21 La fidélité au texte source peut tourner à l'excès quand il semble qu'il s'agisse plus d'une fidélité à la forme qu'au sens. Ainsi, dans la version française, « cédant aux instances de Don Bruno Crespi » se veut une traduction de "a instancias de don Bruno Crespi »; mais alors que cette forme espagnole est d'usage courant, la traduction française semble plus littéraire (ce qui n'est pas le cas des versions brésilienne et anglaise).

Et que dire de la traduction française de «en una película » par «dans un certain film»? Là, c'est par une infidélité manifeste au texte original qu'est obtenu le même effet de préciosité stylistique, qui complique le texte et sa lecture et que l'on ne retrouve pas dans les autres versions ${ }^{7}$.

\subsection{Choix syntaxiques}

23 Un autre phénomène concerne des choix syntaxiques dans certaines traductions, qui ne semblent pas correspondre à l'original en espagnol et qui créent, là encore, des distorsions de lisibilité. Ainsi, en espagnol, l'extrait commence par :

Deslumbrada por tantas y tan maravillosas invenciones [...]

Il semble que la traduction du texte du PISA pour le Brésil (qui est ici celle de Zagury également), comme les versions anglaise et arabe, sont assez conformes.

Mais pourquoi y a-t-il une postposition du deuxième adjectif dans la version française (celle de la traduction des Durand comme celle du PISA) :

Éblouis par tant d'inventions, et si merveilleuses [...]

Cela ne crée-t-il pas une lecture plus difficile qu'une version qui aurait, comme dans l'original, associé deux adjectifs sans les séparer par le nom qu'ils qualifient (par exemple «si nombreuses et si merveilleuses ») ?

26 À cette postposition, qui complique indument la lecture du texte, répond un choix d'antéposition qui aboutit au même effet: la version française traduit ainsi « las imágenes vivas » par « les vivantes images », quand le tout simple « les images vivantes » convenait 
mieux, à tout point de vue (celui de la traduction comme de la compréhension) et s'accordait mieux aux autres versions du PISA, dont les traductions sont plus conformes à la logique des langues cibles.

Notons également, en français, une utilisation récurrente de pronoms relatifs composés, peu usités dans le langage courant: duquel (deux fois), laquelle. Pour ces exemples, la traduction française est la seule de notre corpus à choisir une forme peu usuelle (qui se trouve dans la traduction des Durand).

Comme on le voit, ces choix lexicaux et syntaxiques sont bien des illustrations de cette traduction «trop libre ou trop littéraire » (OECD, 2002, p. 59 ; nous traduisons) qu'il faut éviter selon les principes du PISA que nous avons décrits. Nous pourrions prendre d'autres exemples, mais venons-en à un troisième phénomène, plus problématique.

\subsection{Choix discursifs}

Jusque-là, les choix de traduction pouvaient avoir des incidences sur la lisibilité, ce qui est gênant mais peut-être sans conséquence importante pour la compréhension. Plus graves, dans la logique d'une évaluation internationale des compétences en lecture, sont les choix qui peuvent rejaillir sur la compréhension du texte en lien avec les questions posées. C'est le cas, par exemple, de la traduction française de ce passage (empruntée à celle des Durand) :

[...] porque un personaje muerto y sepultado en una película y por cuya desgracia se derramaron lágrimas de aflicción, reapareció vivo y convertido en árabe en la película siguiente.

[...] à cause d'un personnage mort et enterré dans un certain film, sur le malheur duquel on versa des larmes amères, et qui reparut bien vivant et transformé en Arabe dans le film suivant.

Cette traduction n'est pas en soi problématique, mais elle rend la causalité en français différente de celle du texte espagnol, ce qui peut avoir une incidence sur la réponse donnée à la première question de l'exercice du PISA :

Dans les films, quel est l'élément qui a mis en colère les gens de Macondo?

Que característica dos filmes causou indignação no povo de Macondo?

31 L'élément en question n'est pas un personnage mort qui reparait (réponse qu'induit la traduction proposée), mais le fait qu'un personnage mort reparaisse (réponse qu'aurait induite une traduction plus conforme au texte espagnol).

La différence de causalité aurait pu pourtant être rendue similaire à celle du texte source par une traduction plus adaptée, plus proche des autres versions, qui aurait commencé par « parce qu'un personnage [...] ».

La question des personnages ressurgit dans des choix de traduction étonnants, quand on se rend compte que les versions diverses du PISA ne prennent pas les mêmes décisions pour traduire un même mot dans le texte d'origine. Ainsi, dans le texte original en espagnol (et dans la version espagnole du PISA), il est question de personaje à deux reprises: à propos d'un personaje muerto et à propos des vicisitudes de los personajes. Or cette répétition d'un même mot n'est pas rendue de la même manière selon les versions, comme le montre ce tableau : 
Tableau 1. - Traductions de personaje.

\begin{tabular}{|l|l|l|l|l|l|}
\hline Version originale & $\begin{array}{l}\text { Anglais } \\
(\mathrm{OECD}, 2009)\end{array}$ & $\begin{array}{l}\text { Arabe } \\
\text { (Tunisie) }\end{array}$ & $\begin{array}{l}\text { Espagnol } \\
\text { (Espagne) }\end{array}$ & $\begin{array}{l}\text { Français } \\
\text { (OCDE, 2012) }\end{array}$ & $\begin{array}{l}\text { Portugais } \\
\text { (Brésil) }\end{array}$ \\
\hline personaje muerto & character & personaje & personnage & personagem \\
\hline vicisitudes de los personajes & actors & المّّخصيّات & personajes & personnages & atores \\
\hline
\end{tabular}

Seules les versions espagnole et française du PISA utilisent dans les deux cas le même mot. Les autres choisissent d'utiliser deux mots, respectivement proches de personnage et d'acteur $^{8}$ de façon quasiment interchangeable, ce qui les rapproche d'une certaine manière, du point de vue des habitants de Macondo... Mais, surtout, nous verrons plus loin que la quatrième question de l'unité oblige à la distinction entre les deux mots !

Ces options de traduction qui compliquent le rapport à la fiction, pourtant objet du questionnaire d'évaluation', s'observent encore dans cette partie finale du texte où le mot " fingidas ", que l'on trouve dans la phrase conclusive de l'extrait, n'est pas traduit dans les versions en français et arabe (sans doute réalisée à partir de la première), alors que les autres extraits du corpus maintiennent cette précision.

Plus surprenant encore est la traduction en arabe de " habían sido víctimas de un nuevo y aparatoso asunto de gitanos » :

$$
\text { اعتبر أكثر النّاس أنّ الغجـر تحيّلوا عليهم مجدّدا }
$$

Le raccourci qu'elle propose peut être rendu ainsi en français : "de nouveau, les gitans leur ont joué un mauvais tour ». L'expression éloigne cette phrase, encore plus que dans les autres versions, de sa fonction référentielle par rapport aux évènements du roman, où les gitans apparaissent dès la première page comme des personnages introduisant des inventions dans Macondo et semble ainsi ancrer le propos dans un préjugé à l'égard des gitans...

\section{La traduction au-delà du texte}

Nous avons abordé, jusqu'à maintenant, la question du traitement du texte lui-même. Mais la question de la traduction se pose aussi pour ce qui est du dispositif d'évaluation, à savoir les questions posées, le guide de correction donné aux correcteurs des tests et les dimensions pratiques de la passation des tests. Là encore, sans qu'il soit possible d'être exhaustif, voici quelques-unes des interrogations qui nous sont venues à la lecture des textes du PISA.

\subsection{Le chapeau de présentation du texte}

Dans le chapeau, Macondo est présenté en anglais comme «town» (OECD, 2009, p. 174), " petite ville» en français (OCDE, 2012, p. 166), قرية ماكندو en arabe (qu'on peut rendre par « village »), « pueblo » en espagnol et «cidade » en portugais. Sans nous arrêter sur ses différences sémantiques, il est plus intéressant de signaler la curieuse vision du monde que charrient les mots employés par le PISA dans son commentaire de l'exercice pour 
désigner les habitants de Macondo: «unsophisticated villagers» (OECD, 2009, p. 176), « simples villageois » (OCDE, 2012, p. 168)...

Observons un fait curieux : dans notre corpus, seule la version française propose le même terme imaginaire dans le texte (" êtres imaginaires ») et dans le chapeau qui le présente aux élèves (" petite ville imaginaire»). Dans les autres versions (non reproduites en annexe pour des raisons de place), on distingue « imaginarios » et « ficticio » (en espagnol), « imaginary» et "fictional » (en anglais), « imaginários » et «fictícia » (en portuguais), et, pour finir (en arabe) وهميّين (qui peut se rendre en français par fictif, chimérique, fantomatique) et الخياليّة (qui est sémantiquement proche du français imaginaire).

Cela crée une distorsion, entre les versions, dans la difficulté ou la facilité qu'induit ou non la répétition d'un même terme dans le texte support et dans les questions qui sont posées sur lui (comme le montrent, à propos d'autres évaluations internationales, GuérinPace \& Blum, 1999, p. 281).

\subsection{Les questions posées sur le texte}

Les questions posées aux élèves sont également un sujet d'interrogation. Prenons la première question, dont voici les versions anglaise et française :

What feature of the movies caused the people of Macondo to become angry? (OECD, 2009, p. 174)

Dans les films, quel est l'élément qui a mis en colère les gens de Macondo? (OCDE, 2012, p. 166)

La formulation même de la question en français est curieuse : le syntagme les films est complément circonstanciel, placé en tête de phrase alors qu'il est, dans toutes les autres versions, le complément du nom de l'objet à identifier (et il est placé après ce dernier) : il n'est pas sûr que le sens soit différent ni que la compréhension change, mais la question se pose de savoir pourquoi ce choix stylistique, au demeurant assez curieux, a été fait alors que la même construction était possible en français : "Quel est l'élément des films qui a mis en colère les gens de Macondo?»

La question 3 est un QCM, que voici reproduite en anglais et en français :

At the end of the passage, why did the people of Macondo decide not to return to the movies?

(OECD, 2009, p. 176)

À la fin du passage, pourquoi les gens de Macondo ont-ils choisi de ne plus se rendre

au cinéma ? (OCDE, 2012, p. 168)

Des quatre réponses proposées par le QCM, intéressons-nous aux deux dernières, que nous reproduisons, ici encore, en anglais :

C. They wanted to save their emotions for real-life occasions.

D. They were seeking emotional involvement, but found the movies boring, unconvincing

and of poor quality. (OECD, 2009, p. 176)

et en français :

C. Ils préféraient garder leurs émotions pour des occasions de la vie réelle.

D. Ils cherchaient à s'impliquer émotionnellement, mais ils ont trouvé les films

ennuyeux, peu convaincants et de mauvaise qualité. (OCDE, 2012, p. 168)

On le voit par les mots soulignés en gras, ces deux réponses font référence à l'émotion, par l'usage du nom (emoções, emociones, emotions, émotions) ou d'un dérivé adjectival ( emocional, emotional) ou adverbial (emocionalmente, émotionnellement); or, à la fin du texte (où doit se trouver la réponse à la question, comme il est précisé dans cette dernière), si on trouve un mot de la famille de «émotion » dans les versions anglaise (" 
the emotional outburst of the audience ») et brésilienne (" arrebatamentos de emoção da plateia $»)$, ce n'est pas le cas dans les versions espagnole ("los desbordamientos pasionales del público ») ou française (« ces débordements passionnels du public ») ${ }^{10}$.

La question suivante est également un QCM, dont voici les versions anglaise et française :

Who are the "imaginary beings" referred to in the last line of the passage? (OECD, 2009,

p. 177)

Qui sont les «êtres imaginaires » dont on parle à la dernière ligne du texte ? (OCDE,

2012, p. 168)

Dans la troisième et la quatrième modalités de réponse qu'elle propose, cette question du test oblige à faire la distinction entre deux mots pour désigner les personnages et les acteurs :

Tableau 2. - Traductions de personajes et actores.

\begin{tabular}{|l|l|l|l|l|l|}
\hline & $\begin{array}{l}\text { Anglais } \\
\text { (OECD, 2009) }\end{array}$ & $\begin{array}{l}\text { Arabe } \\
\text { (Tunisie) }\end{array}$ & $\begin{array}{l}\text { Espagnol } \\
\text { (Espagne) }\end{array}$ & $\begin{array}{l}\text { Français } \\
\text { (OCDE, 2012) }\end{array}$ & $\begin{array}{l}\text { Portugais } \\
\text { (Brésil) }\end{array}$ \\
\hline Réponse C & characters & ممثـلون & actores & acteurs & atores \\
\hline Réponse D & actors & personnages & personagens \\
\hline
\end{tabular}

47 Le problème est que, dans le texte support, on l'a vu plus haut, certaines versions (anglaise, arabe, portugaise) proposent bien les deux mots, alors que les versions française et espagnole n'en proposent qu'un (personnage, personaje). Il est assez facile de prévoir que cela aura quelque incidence sur la perception de la question.

\subsection{L'environnement de passation des tests}

Au-delà du matériau directement donné aux élèves qui passent le test, il serait sans doute utile de comparer aussi ce que l'on peut appeler l'environnement du test. Prenons le cas du guide de correction et de codage destiné aux personnes chargées de coder les réponses aux autres questions que les QCM. La procédure de traduction pour les guides nationaux de correction des réponses aux questions ouvertes du test est plus simple que celle que nous avons décrite pour le test lui-même (OECD, 2016b, p. 6). Certains pays ont pu notamment (ibid.) faire le choix de ne produire qu'une seule version nationale du guide bien qu'il y ait différentes langues d'enseignement (par exemple en espagnol en Espagne), tandis que d'autres n'ont pas réalisé de traduction du guide et ont utilisé la version anglaise (en Suède notamment) ou française (en Tunisie, par exemple), même si les élèves répondaient dans une langue différente. Si toute personne participant au codage des réponses au test doit avoir participé à des sessions de formation (ou d'entrainement) mise en œuvre par chaque institution responsable de la mise en œuvre nationale du programme (OECD, 2002, p. 75 et suiv.), la question se pose des effets des choix en matière de traduction du guide de correction et de codage réalisé, qui va devenir la «matière première" des résultats du PISA. Ce qui est en jeu est la nature du guidage de la perception des réponses des élèves aux questions ouvertes, fondamentale dans l'entreprise même duPISA. Cette question de la perception des réponses par des correcteurs (abordée par Robin [2002], dans son analyse d'un guide de correction d'une 
autre unité du PISA et des corrections effectivement faites aux tests) demanderait d'importantes investigations ${ }^{11}$.

Cette question engage à envisager d'autres aspects de l'environnement et du design de l'administration des tests, qui dépassent la question stricte de la traduction mais qui, comme cette dernière, concerne le problème des langues. C'est le cas par exemple des conditions matérielles de leur passation ou celle du contexte linguistique des élèves, par exemple quand plusieurs langues sont langues d'enseignement, ou encore quand les langues d'enseignement ne sont pas les langues d'usage d'une grande partie de la population. Qu'on songe par exemple (cf. Belaid Mhirsi, à paraitre) au problème qui se pose pour l'usage de l'arabe en Tunisie (et ailleurs) : d'une part les élèves tunisiens sont peu familiarisés avec le clavier en langue arabe (l'informatique, étudiée en français dès le grade 7 - élèves de 12 à 13 ans - est très peu utilisée pour les matières enseignées en arabe) et d'autre part la majorité des élèves de 15 ans participant au PISA sont au lycée (en grade 10), niveau à partir duquel les matières scientifiques sont enseignées en français. Lors du PISA 2015, ces élèves ont pu avoir le choix de répondre en arabe ou en français à des questions posées en arabe - sachant que les correcteurs disposaient, comme nous venons de le préciser, d'un guide de correction en français, c'est-à-dire dans une autre langue que celle des questions posées... Bien sûr, cela dépasse la question de l'évaluation du PISA et engage plus généralement la problématique linguistique des pays de langue arabe où la langue d'enseignement est l'arabe standard ${ }^{12}$. Mais cet exemple permet de voir néanmoins que ces problèmes de traduction dans le cadre du PISA renvoient plus largement à des enjeux majeurs concernant les langues dans les systèmes éducatifs et au-delà.

\section{Conclusion}

De telles questions laissent apparaitre combien la question des langues, fût-elle prise en compte par les protocoles de traduction du PISA, contient de nombreuses occasions de pièges. D'autant que toutes les précautions ne sont pas prises pour appliquer au mieux les principes de traduction du PISA: on a pu voir les nombreuses disparités entre les traductions, tant au niveau des mots que de la syntaxe ou du discours, dont on peut supposer qu'elles créent autant de disparités au niveau de la réception du texte et de l'engagement du lecteur. Il est d'ailleurs curieux que l'unité " Macondo », non réutilisée depuis sa mise en œuvre lors du premier cycle de l'année 2000 ait été proposée pour un essai de terrain en $2009^{13}$, sans révisions de la traduction; il est plus curieux encore que, sans mention des nombreux problèmes de traduction que nous avons identifiés, elle ait pu être donnée comme exemple représentatif par le PISA dans son cadre d'évaluation de 2009 (OECD, 2009; OCDE, 2012) - ce qui, du reste, a justifié notre choix d'utiliser cette unité pour notre analyse des distorsions entre les principes affichés par le PISA et leur mise en œuvre effective.

51 Il faudrait d'autres investigations pour savoir si les observations que nous avons faites ici à propos d'un texte de fiction sont valables pour d'autres types d'évaluation: sans doute la traduction d'un texte littéraire pose-t-elle des problèmes spécifiques et il n'est pas impossible que l'objet même de l'évaluation - la compréhension - engendre des effets plus visibles que dans les autres domaines du test (culture scientifique, culture mathématique). Mais la plupart de nos observations nous semblent pouvoir, sous réserve de vérification, être généralisées, notamment celles qui portent sur la variation (selon les 
traductions) des cohérences internes au discours et des relations entre les signes et leurs référents.

Certes, la sophistication de la méthodologie psychométrique du PISA permet d'espérer que les biais de traduction peuvent être négligés, d'un point de vue statistique Mais nul ne pourrait songer compenser par la statistique les défauts de ce qui est précisément au fondement de l'évaluation de la compréhension : le texte et sa compréhension. De fait, la pertinence d'une méthode de traitement des données, aussi sophistiquée soit-elle, repose sur la qualité des données elles-mêmes, donc, in fine, sur celle du matériel de test ; or, dans une comparaison internationale, cette qualité repose notamment sur la fiabilité des traductions, ce qui explique l'extrême attention du PISA sur le processus de traduction. Et c'est, en retour, ce qui nous amène à interroger la fiabilité du processus d'évaluation, quand on voit, sur l'exemple que nous avons analysé, les importants problèmes qui résultent de la mise en œuvre de ce processus de traduction.

L'approche du PISA, comme le dit Chartier, « conduit à accréditer comme évidente l'idée que les normes d'un temps et d'un espace, celui des pays de l'OCDE, doivent avoir une validité universelle. C'est l'hypothèse (ou le préjugé) de tous les statisticiens qui dressent l'atlas de l'alphabétisation mondiale, en faisant du savoir-lire une donnée invariante » (2007, p. 277). Cette illusion de pouvoir rendre compte des compétences de la lecture sans tenir compte des contraintes contextuelles du temps et de l'espace trouve son illustration sans doute la plus manifeste dans la confrontation des langues. Paradoxalement, la conception universaliste du PISA, qui fonde la large diffusion des contenus de ses tests permet, en retour, d'en interroger la pertinence : c'est ce qui pourrait permettre d'affiner davantage l'analyse du programme et de faire évoluer son dispositif d'évaluation.

À cet égard, nous ne croyons pas pouvoir donner des propositions concrètes, pour une raison simple - outre le fait qu'elles auraient peu de chance d'être entendues: c'est qu'elles auraient toute chance d'être conformes aux exigences que le PISA prétend luimême se donner dans son traitement méthodologique des questions de traduction et aux évolutions que le programme apporte à son processus traductionnel (présentées dans la partie 2). Car rappelons que notre objectif était de montrer que le PISA peut ne pas respecter ses propres exigences. Cela s'expliquerait facilement par la complexité de la tâche : mais ce qui reste inexplicable est la dénégation du PISA, qui s'ingénie, contre toute logique scientifique, à ne pas discuter les problèmes effectifs que rencontre la mise en œuvre de son évaluation et les conséquences possibles sur ses résultats.

\section{BIBLIOGRAPHIE}

ABADZI, Helen \& MARTELLI, Marialuisa. (2014, novembre). Efficient Reading for Arab Students: Implications from Neurocognitive Research. Communication présentée au « World Summit of Innovation in Education (WISE) », Doha, Qatar.

ARFFMAN, Inga. (2010). Equivalence of Translations in International Reading Literacy Studies. Scandinavian Journal of Educational Research, 54(1), 37-59. 
BART, Daniel \& DAUNAY, Bertrand. (2016). L'évaluation de la lecture d'un texte littéraire selon le programme PISA : interrogations didactiques. Dans : É. Falardeau, P. Lefrançois, J.-L. Dumortier \& J. Dolz (dir.), L'évaluation dans la discipline français (p. 175-193). Namur : AIRDF.

BART, Daniel \& DAUNAY, Bertrand. (2017, octobre). o discurso do PISA: Interrogações sobre uma interculturalidade de ficção. Conférence donnée à l'Université PUC Minas, Belo Horizonte, Brésil.

BELAID MHIRSI, Chedia. (2015). Enseignement des sciences et des technologies dans le système éducatif tunisien : les défis à relever. Dans S. Kamoun-Chouk (dir.), Sciences et technologies dans les réformes des systèmes éducatifs. Quelles innovations pour demain? (p. 225-246). Tunis : Librairie Beït Al-Hikma.

BELAID MHIRSI, Chedia. (À paraitre). Reliance et Plurilinguisme. Le contexte linguistique tunisien entre déliance et reliance ou la tentation du monolinguisme. Actes du colloque « La Reliance. De la sociologie à la littérature ", Institut supérieur des sciences humaines et sociales de Tunis, Tunis, Tunisie.

BONNET, Gérard. (2006). Tener presentes las singularidades lingüísticas y culturales en las evaluaciones internacionales de las competencias de los alumnos: ¿una nueva dimensión para PISA? Revista de Educación, n. ${ }^{\circ}$ extraordinario, 91-109.

CHARTIER, Anne-Marie. (2007). L'école et la lecture obligatoire. Paris : Retz.

CNIPRE (Centre national d'innovation pédagogique et de recherche en éducation ()). (2009). ماكندو Macondo). Tunis : CNIPRE.

DAUNAY, Bertrand \& BART, Daniel. (2016, mai). Le PISA : quelques paradoxes d'une évaluation des compétences en littératie. Communication invitée au séminaire « Enfoque Letras 2016 », Université PUC Minas, Belo Horizonte, Brésil.

GoLDSTEIN, Harvey. (2008). Comment peut-on utiliser les études comparatives internationales pour doter les politiques éducatives d'informations fiables? Revue française de pédagogie, 164, 69-76.

GRISAY, Aleta. (2003). Translation Procedures in OECD/PISA 2000 International Assessment. Language Testing, 20(2), 225-240.

GRISAY, Aleta. (2007). The Challenge of Adapting PISA Materials into Non Indo-European Languages. Some Evidence from a Brief Exploration of Language Issues in Chinese and Arabic. Doc: TAG(0801). Paris : OECD/PISA. Disponible en ligne sur <www.aspe.ulg.ac.be/grisay/fichiers/PISA07.pdf>.

GriSAY, Aleta, De Jong, John H. A. L., GEBHARDT, Eveline, BEREZner, Alla \& HAlLEUX-MonSEUR, Béatrice. (2007). Translation Equivalence across PISA Countries. Journal of Applied Measurement, 8 (3), 249-266.

GUÉRIN-PACE, France \& BLUM, Alain. (1999). L'illusion comparative. Les logiques d'élaboration et d'utilisation d'une enquête internationale sur l'illettrisme. Population, 54(2), 271-302.

INEP (Instituto Nacional de Estudos e Pesquisas Educacionais Anísio Teixeira). (2009). Itens: PISA 2009. INEP : PISA. Disponible en ligne sur <http://portal.inep.gov.br/internacional-novopisa-itens> (consulté le 14 mai 2016).

MiNISTERIO DE EdUCACIÓN DE ESPAÑA. (2010). La lectura en PISA 2009. Marcos y pruebas de la evaluación. Madrid : Secretaría general técnica.

OCDE. (2012). Le cadre d'évaluation de PISA 2009. Paris: Éditions OCDE. Disponible en ligne sur < www.oecd.org/pisa/pisaproducts/49539189.pdf>. 
OECD. (2002). PISA 2000 Technical Report, edited by Ray Adams \& Margaret Wu. Paris : OECD

Publishing.

OECD. (2004). PISA 2006 Translation and Adaptation Guidelines. Paris : OECD Publishing.

OECD. (2005). PISA 2003 Technical Report. Paris : OECD Publishing.

OECD. (2009). Assessment Framework. Key Competencies in Reading, Mathematics and Science. Paris :

OECD Publishing.

OECD. (2012). PISA 2009 Technical Report. Paris : OECD Publishing.

OECD. (2014). PISA 2012 Technical Report. Paris : OECD Publishing.

OECD. (2016a). PISA 2015 Technical Report. Chapter 3: Context Questionnaire Development. Paris : OECD

Publishing.

OECD. (2016b). PISA 2015 Technical Report. Chapter 5: Translation and Verification of the Survey Material . Paris : OECD Publishing.

OECD. (2016c). PISA 2018 Translation and Adaptation Guidelines. Paris : OECD Publishing.

PuchHAmmer, Markus. (2007). Language-Based Item Analysis. Problems in Intercultural

Comparisons. Dans S. T. Hopmann, G. Brinek \& M. Retzl (dir.), PISA zufolge PISA - PISA According to PISA (p. 127-137). Berlin-Wien : Lit Verlag.

RÉMOND, Martine. (2005). Regards croisés sur les évaluations institutionnelles. Repères, 31, 113-140.

RoBin, Isabelle. (2002). L'enquête PISA sur les compétences en lecture des élèves de 15 ans : trois biais culturels en question. VEI Enjeux, 129, 65-91.

ROCHER, Thierry. (2003). La méthodologie des évaluations internationales des compétences. Psychologie et Psychométrie, 24(2/3), 117-146.

SJøBERG, Svein. (2016). OECD, PISA, and Globalization: The Influence of the International Assessment Regime. Dans C. H. Tienken \& C. A. Mullen (dir.), Education Policy Perils. Tackling the Tough Issues (p. 102-133). Londres : Routledge.

\section{ANNEXES}

\section{CORPUS}

\section{ANNEXE 1. - Extrait de l'unité « Macondo » en français}

(OCDE, 2012, p. 166-171)

Éblouis par tant d'inventions, et si merveilleuses, les gens de Macondo ne savaient par où commencer à s'étonner. Ils passaient des nuits blanches à contempler les pâles ampoules alimentées par le groupe électrogène qu'avait rapporté Aureliano le Triste lors du second voyage du train, et à l'obsédant teufteuf duquel on ne s'habitua qu'à la longue, péniblement. Ils furent indignés par les vivantes images que le riche commerçant Don Bruno Crespi projetait dans le théâtre aux guichets en gueules de lion, à cause d'un personnage mort et enterré dans un certain film, sur le malheur duquel on versa des larmes amères, et qui reparut bien vivant et transformé en Arabe dans le film suivant. Le public, qui payait deux centavos pour partager les mésaventures des personnages, ne put supporter cette inqualifiable moquerie et brisa tous les sièges. Le maire, cédant aux 
instances de Don Bruno Crespi, dut faire une proclamation publique pour expliquer que le cinéma n'était qu'une machine à illusions, laquelle ne méritait pas ces débordements passionnels du public. À la suite de cette décevante explication, beaucoup estimèrent qu'ils avaient été victimes d'une nouvelle et spectaculaire affaire de gitans, si bien qu'ils choisirent de ne plus se rendre au cinéma, considérant qu'ils avaient assez de leurs propres peines pour aller encore pleurer sur les malheurs d'êtres imaginaires.

Le texte de la page ci-contre est extrait d'un roman. Dans cette partie du récit, on vient d'introduire le chemin de fer et l'électricité dans la petite ville imaginaire de Macondo, et d'ouvrir le premier cinéma.

Référez-vous à ce texte afin de répondre aux questions suivantes.

\section{Question 1}

Dans les films, quel est l'élément qui a mis en colère les gens de Macondo?

\section{Question 3}

À la fin du passage, pourquoi les gens de Macondo ont-ils choisi de ne plus se rendre au cinéma?

A. Ils voulaient s'amuser et se distraire, mais ils ont trouvé que les films étaient réalistes et déprimants.

B. Ils n'avaient pas les moyens de payer le prix des billets.

C. Ils préféraient garder leurs émotions pour des occasions de la vie réelle.

D. Ils cherchaient à s'impliquer émotionnellement, mais ils ont trouvé les films ennuyeux, peu convaincants et de mauvaise qualité.

\section{Question 4}

Qui sont les « êtres imaginaires » dont on parle à la dernière ligne du texte?

A. Des fantômes.

B. Des inventions de champ de foire.

C. Des personnages des films.

D. Des acteurs.

\section{Question 5}

Êtes-vous d'accord avec le jugement final des gens de Macondo à propos de l'intérêt du cinéma ? Expliquez votre réponse en comparant votre attitude et la leur à l'égard des films.

\section{ANNEXE 2. - L'extrait en anglais, en arabe, en espagnol et en portugais}

Version en anglais (OECD, 2009, p. 174)

Dazzled by so many and such marvellous inventions, the people of Macondo did not know where their amazement began. They stayed up all night looking at the pale electric bulbs fed by the plant that Aureliano Triste had brought back when the train made its second trip, and it took time and 
effort for them to grow accustomed to its obsessive toom-toom. They became indignant over the living images that the prosperous merchant Don Bruno Crespi projected in the theatre with the lion-head ticket windows, for a character who had died and was buried in one film, and for whose misfortune tears of affliction had been shed, would reappear alive and transformed into an Arab in the next one. The audience, who paid two centavos apiece to share the difficulties of the actors, would not tolerate that outlandish fraud and they broke up the seats. The mayor, at the urging of Don Bruno Crespi, explained by means of a proclamation that the cinema was a machine of illusions that did not merit the emotional outburst of the audience. With that discouraging explanation many felt that they had been the victims of some new and showy gypsy business and they decided not to return to the movies, considering that they already had too many troubles of their own to weep over the acted-out misfortunes of imaginary beings.

Version en arabe (Tunisie, CNIPRE, 2009)

انبهر سكّان ماكندو بالكمّ الهائل من الاختراعات العجيبة حِّى احتاروا أيّها أكثر مَدْعَاة

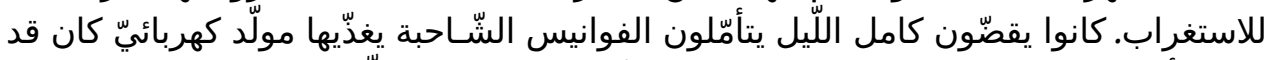

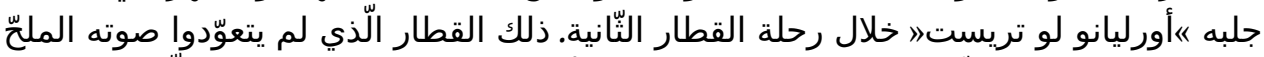

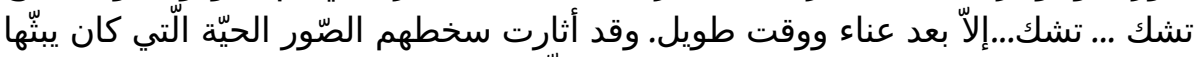

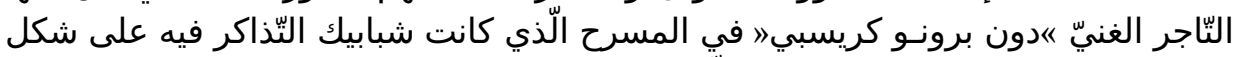

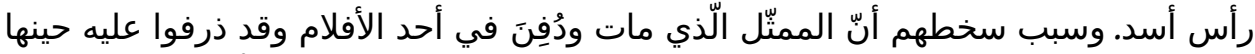

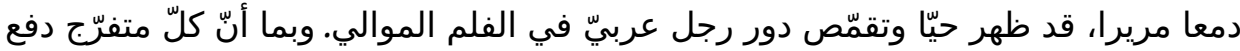

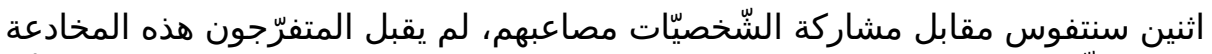

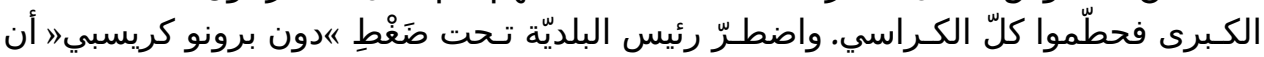

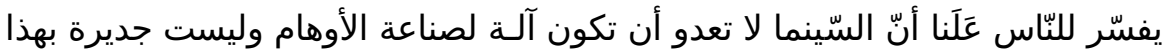

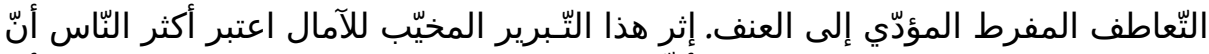

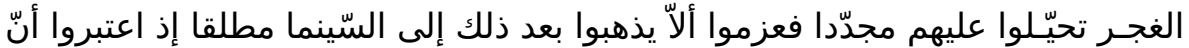

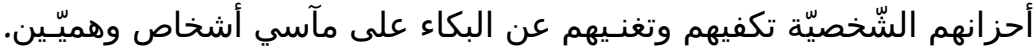

Version en espagnol (Espagne, Ministerio de educación de España, 2010, p. 148)

Deslumbrada por tantas y tan maravillosas invenciones, la gente de Macondo no sabía por dónde empezar a asombrarse. Se trasnochaban contemplando las pálidas bombillas eléctricas alimentadas por la planta que llevó Aureliano Triste en el segundo viaje del tren, y a cuyo obsesionante tumtum costó tiempo y trabajo acostumbrarse. Se indignaron con las imágenes vivas que el próspero comerciante don Bruno Crespi proyectaba en el teatro con taquillas de bocas de león, porque un personaje muerto y sepultado en una película y por cuya desgracia se derramaron lágrimas de aflicción, reapareció vivo y convertido en árabe en la película siguiente. El público que pagaba dos centavos para compartir las vicisitudes de los personajes, no pudo soportar aquella burla inaudita y rompió la silletería. El alcalde, a instancias de don Bruno Crespi, explicó mediante un bando que el cine era una máquina de ilusión que no merecía los desbordamientos pasionales del público. Ante la desalentadora explicación, muchos estimaron que habían sido víctimas de un nuevo y aparatoso asunto de gitanos, de modo que optaron por no volver a ir al cine, considerando que ya tenían bastante con sus propias penas, para llorar por fingidas desventuras de seres imaginarios.

Version en portugais (Brésil, INEP, 2009)

Deslumbrado com tantas e tão prodigiosas invenções, o povo de Macondo não sabia onde começava seu assombro. Ficavam acordados toda a noite com os olhos arregalados, fixos nas lâmpadas elétricas alimentadas pelo gerador que Aureliano Triste trouxera quando da segunda viagem do trem, e levou tempo e custou-lhes esforço para se acostumarem ao seu incessante tum-tum. Ficaram indignados com as imagens vivas que o próspero comerciante Dom Bruno Crespi projetava 
no teatro decorado com cabeças de leão sobre os guichês, pois um personagem que morria e era enterrado em um filme, por cujos infortúnios haviam sido derramadas lágrimas de aflição, tornava a aparecer vivo e transformado em árabe no outro. A plateia, que pagava dois centavos por cabeça para compartilhar das desventuras dos atores, não tolerou esse tipo de fraude inconcebivel e quebrou os assentos. 0 prefeito, cedendo às instâncias de Dom Bruno Crespi, explicou através de uma proclamação pública que o cinema era uma máquina de ilusões que não merecia tais arrebatamentos de emoção da plateia. Após essa explicação desanimadora, muitos acreditaram que tinham sido vítimas de um novo e espetacular negócio de ciganos e decidiram não mais voltar ao cinema, considerando que já tinham problemas suficientes para chorar pelos dramas representados por seres imaginários.

\section{NOTES}

1. Cf. par exemple les travaux - issus de cadres théoriques divers - de Arffman (2010), Bonnet (2006), Goldstein (2008), Guérin-Pace \& Blum (1999), Puchhammer (2007), Rémond (2005), Sjøberg (2016).

2. Plutôt que d'exercices, le PISA parle d'unités. Dans ce programme, chaque unité - pourvue d'un titre - est construite autour d'un «stimulus » (texte, graphique, etc.) auquel se rapportent une ou plusieurs questions (items) accompagnées d'un guide de correction (OECD, 2014, p. 36).

3. Le PISA, régulièrement, rend publiques certaines unités ou certaines parties d'unités, qui ne sont plus, une fois divulguées, utilisées dans les tests. C'est ce que le PISA désigne par «Items libérés » (« Released items »).

4. Nous empruntons les versions anglaise et française à l'Assessment Framework (OECD, 2009) et au Cadre d'évaluation (OCDE, 2012) du cycle PISA 2009 ; nous utilisons la version arabe donnée en Tunisie, la version espagnole en Espagne, la version portugaise au Brésil. Ce corpus de versions (qu'on trouvera en annexe 2), a été construit en fonction de rencontres de travail sur le PISA entre collègues originaires de pays différents, et permet d'explorer des problèmes de traduction qui se posent dans un programme se décrivant comme exemplaire en la matière, mais ne vise pas de représentativité particulière par rapport à l'ensemble du PISA.

5. Pour une première approche des problèmes de traduction de cette unité, cf. Bart et Daunay (2016, 2017), Daunay et Bart (2016). Nous voudrions ici remercier Benoit Deslandes et Michèle Falip-Deslandes pour leur lecture attentive de l'article et leurs conseils avisés.

6. Il était plus délicat de parler de centime, qui n'existe pas dans la monnaie locale, même si le mot, via le français, peut n'être pas inconnu, ce qui explique que ce soit le choix fait par la traduction commerciale de Almani.

7. Précisons que dans le même document de l'OCDE (2012), on trouve une autre version en français que celle que nous analysons (annexe 1) et qui propose la traduction «dans un film » (p. 47).

8. Il faut noter que les termes arabes inversent la valeur sémantique des mots des autres versions, الممثّل se rendant mieux par « acteur » et الشّخصيّات par «personnages »...

9. Voir la question 5 en annexe 1: "Êtes-vous d'accord avec le jugement final des gens de Macondo à propos de l'intérêt du cinéma ? Expliquez votre réponse en comparant votre attitude et la leur à l'égard des films. »

10. Le cas de l'arabe est encore différent, car les modalités de réponse $\mathrm{C}$ et $\mathrm{D}$, contrairement aux autres versions, n'utilisent pas un mot de la même famille, et aucun de ces mots ne correspond à un des mots de la dernière phrase du texte...

11. C'est ce qui a amené Daniel Bart et Bertrand Daunay, avec Juliana Assis et d'autres collègues de l'université PUC Minas de Belo Horizonte, à monter un projet de recherche (en cours) qui 
interroge cette perception par des enseignants, en dehors des protocoles du PISA : «Desafios interculturais de avaliações e comparações internacionais em educação: olhares cruzados BrasilFrança ».

12. On sait que le choix même d'enseigner en arabe standard certaines disciplines scientifiques dans tout l'enseignement de base tunisien est discuté (Belaid Mhirsi, 2015). Voir par ailleurs Grisay (2007, p.6), qui évoque une diglossie en langue arabe dans un paragraphe intitulé "Standard Arabic vs spoken Arabic »; pour une réflexion sur les problèmes de lecture dans les pays arabes et les performances de ces derniers dans les études comparatives internationales, voir Abadzi et Martelli (2014).

13. Précisons qu'à chaque édition du programme PISA, l'un des trois principaux domaines d'évaluation (compréhension de l'écrit, culture mathématique, culture scientifique) est constitué comme domaine majeur du test. Le domaine majeur est évalué par un nombre plus important d'unités et d'items que les deux autres domaines. Lors des PISA 2000, 2009 et 2018, la compréhension de l'écrit a constitué ce domaine majeur.

\section{RÉSUMÉS}

Pour les évaluations scolaires internationales, les questions de traduction du matériel de test constituent un enjeu méthodologique majeur pour éviter toute objection sérieuse à leur visée de comparer, malgré les différences de langues, les niveaux de compétences des élèves. À ce titre, le Programme international de suivi des acquis des élèves (PISA), souvent présenté comme la référence actuelle pour ce type d'évaluations, développe une réflexion d'une grande solidité concernant ses propres démarches de traduction. Pourtant, une analyse détaillée de différentes versions (en anglais, arabe, espagnol, français et portugais) d'un exercice tiré du PISA dans le domaine de la compréhension de l'écrit permet de décrire plusieurs problèmes liés à des choix lexicaux, syntaxiques ou discursifs. Si toute entreprise de traduction est discutable, ce travail critique, ici réalisé au-regard des principes mêmes que s'est donnés le PISA, nous permettra non seulement de discuter les limites des résultats obtenus et de leur comparabilité, mais interrogera plus largement une certaine conception des langues et de la lecture que véhicule cette évaluation internationale de la littéracie des élèves.

For international school assessments, the problems of the translation of the test material, are an important methodological challenge in order to avoid any serious objection to their aim to compare, despite the differences in languages, the skill levels of students. It's the reason why the Program for International Student Assessment (PISA), often considered as the current reference for this type of evaluation, develops a very solid reflection concerning its own translation processes. However, a detailed analysis of different versions (in English, Arabic, Spanish, French and Portuguese) of a PISA exercise in the field of reading literacy can uncover several problems related to lexical, syntactic or discursive choices and consider their consequences. If any translation can indeed be questionable, this critical work, that was done by taking into account the very principles adopted by the PISA, will enable us not only to discuss the limits of the results obtained and their comparability, but will also question a certain way of thinking the language and the reading in this international assessment of student literacy. 
INDEX

Mots-clés : évaluations internationales, PISA, traduction, langues, littéracie, approche critique

Keywords : international assessments, PISA, translation, languages, literacy, critical approach

\section{AUTEURS}

DANIEL BART

Université de Lille, Théodile-CIREL

BERTRAND DAUNAY

Université de Lille, Théodile-CIREL

CHEDIA BELAID MHIRSI

Consultante en éducation, doctorante à l'Université Sorbonne Nouvelle (DILTEC) et l'Université de la Manouba (Tunisie) 